\title{
Suizid - ein Thema für die Psychoanalyse
}

\author{
Paul Götze • Ulrich Lamparter
}

Online publiziert: 7. Mai 2020

(C) Springer Medizin Verlag GmbH, ein Teil von Springer Nature 2020

Der Selbstmord ist ein Ereignis der menschlichen Natur, welches, mag auch darüber schon soviel gesprochen und gehandelt sein als da will, doch einen jeden Menschen zur Teilnahme fordert, in jeder Zeitepoche wieder einmal verhandelt werden muß. (Goethe 1964)

Dieses unter Suizidologen sehr bekannte Zitat Goethes ist zeitlos und verpflichtend zugleich. Damit verbunden sind ethische, philosophische, soziologische, theologische und kulturhistorische Grundfragen ersten Ranges; sie reichen bis in die Zeiten Sokrates' (Platon 1990) und früher zurück.

Während suizidales Denken, Erleben und Handeln in diesem Sinne als ein allgemein menschliches Phänomen ubiquitär und zeitlos erscheint, sind die Beurteilung der Suizidalität und damit sowohl die gesellschaftliche als auch die individuelle Haltung zu einem suizidalen Mitmenschen immer wieder einem epochalen Wandel unterworfen. Sie stehen in Abhängigkeit vom jeweils vorherrschenden Zeitgeist, in dem die Einstellung zur Frage der Suizidalität durchaus aber auch sehr widersprüchlich sein konnte und auch heute noch sehr uneinheitlich erscheint. So stehen sich heute - vereinfacht formuliert - drei wertorientierte Positionen gegenüber:

- Der Suizid ist ohne Ausnahme zu verurteilen.

- Der Suizid ist abzulehnen, nur in Ausnahmefällen aber durchaus zu rechtfertigen.

- Der Suizid steht in der freien Entscheidung des einzelnen Menschen.

Entsprechend wird von Selbstmord, Suizid, Selbsttötung oder von Freitod gesprochen. In der diagnostisch und therapeutisch orientierten Suizidologie wird wegen seines wertneutralen Verständnisses der Begriff Selbsttötung sowie im internationalen und wissenschaftlichen Sprachgebrauch der Begriff Suizid verwendet.

PD Dr. U. Lamparter ( $ه)$

Rothenbaumchaussee 71, 20148 Hamburg, Deutschland

E-Mail: lamparter@uke.uni-hamburg.de 
In weitgehender Übereinstimmung sprechen wir heute bei Suizidalität von einer Denk-, Erlebens- und Verhaltensweise, die ein bestimmtes psychisches Leiden ausdrückt, eine Bewältigung vom Betroffenen selbst aber nicht möglich erscheinen lässt und überwiegend bewusst oder auch unbewusst auf Fremdhilfe ausgerichtet ist. Nur in diesem Sinne besitzt Suizidalität als „Befindlichkeit“ und Befund sowohl subjektiv als auch objektiv Krankheitswert, ohne jedoch den Kriterien einer Krankheitsentität zu entsprechen. Mit diesem Verständnis ist therapeutisches Handeln verpflichtend.

Die psychoanalytischen Verstehenskonzepte der Suizidalität waren zunächst eher theroriegeleitet und entfalteten sich entlang der allgemeinen psychoanalytischen Theorieentwicklung, insbesondere der Aggressions-, Narzissmus- und der Objektbeziehungstheorien sowie der Selbstpsychologie. Es zeigte sich jedoch schon bald, dass bei allem Bemühen es nicht gelang, eine quasi übergreifende einheitliche psychoanalytische Theorie der Suizidalität zu entwickeln. Vielmehr wurden mehr oder weniger lediglich Teilaspekte ausgearbeitet, die sich aber durchaus für das Verständnis der Suizidalität und für psychotherapeutische Handlungsansätze als sehr hilfreich erwiesen. Auch hier zeigt sich: Suizidales Erleben und Verhalten ist eben keine Krankheitsentität.

Schon frühzeitig wurde auf die Bedeutung der Reaktion des Therapeuten auf den Suizid seines Patienten sowie auf die Therapeut-Patient-Interaktion beim Auftreten von Suizidalität während einer noch laufenden psychotherapeutischen Behandlung hingewiesen, im Sinne einer wechselseitigen projektiven Identifikation.

Litman (1965) befragte mehr als 200 Psychotherapeuten kurz nach dem Suizid eines ihrer Patienten. Die Therapeuten äußerten Gefühle wie Betroffenheit, Kummer, Schuld und Depressivität und machten sich nicht selten den Vorwurf angstgeprägter persönlicher Unzulänglichkeit. Zum Teil seien nach Litman unbewusste Identifikationen des Therapeuten mit dem Patienten aufgetreten, die sich zum Beispiel in einer Anfälligkeit des Therapeuten für Unfälle nach dem Patientensuizid geäußert hätten. Verleugnung sei der häufigste Abwehrmechanismus der Therapeuten gewesen.

Auch Bloom (1967) fand in Interviews mit Psychotherapeuten, deren Patienten sich suizidiert hatten, dass die meisten Therapeuten mit Verleugnung, Verdrängung und Isolierung von Gefühlen reagierten. Aufzeichnungen über die letzte Zeit der Therapie seien spärlich oder gar nicht mehr vorhanden gewesen. In seinen Schlussfolgerungen erwähnt Bloom, dass eine psychodynamische Interpretation der klinischen Befunde nahelegen würde, dass Feindseligkeit und Abhängigkeit als Übertragungsreaktionen der Patienten bei den Therapeuten ebenfalls Feindseligkeit sowie Zurückweisung als Gegenübertragungsreaktionen im Vorfeld des Suizids hervorrufen würden. Maltsberger und Buie (1974) prägten in diesem Zusammenhang den Begriff des sog. Gegenübertragungshasses. Dem Patienten würde es unbewusst gelingen, durch Übertragungsreaktionen den Narzissmus des Therapeuten anzugreifen, zum Beispiel auf dessen Identifikation mit einem erfolgreichen Therapeuten, wodurch bei diesem unkontrollierter Ärger und Wut ausgelöst und als Gegenübertragungswiderstand ausagiert würden.

Aber nicht nur in therapeutischen Situationen, sondern grundsätzlich muss insbesondere der psychoanalytisch tätige Therapeut, der sich mit suizidalen Patienten auseinandersetzt, eine reflektierte stabile und authentische Haltung zu sich selbst erworben haben. Denn in der Begegnung mit suizidalen Patienten spiegeln unse- 
re negativen Gegenübertragungsgefühle im Sinne einer projektiven Identifikation nicht nur das unerträgliche Erleben des Patienten wider. Auch unsere eigenen unbewussten neurotischen Anteile, das heißt nicht selten unsere abgewehrten und selbst als unerträglich empfundenen unbewussten aggressiven Wünsche und Hassgefühle, unsere Ängste, unsere eigene potenzielle Kränkbarkeit und unsere Schuldgefühle werden mobilisiert. Dies geht bis hin zu eigenen suizidalen Fantasien und Befindlichkeiten im Sinne der Reaktivierung unerträglicher konfliktbesetzter Selbst- und Objektbeziehungsanteile. Lange zurückliegende Todes- und andere Objektverlusterfahrungen können so erst in der psychotherapeutischen Übertragungs- und Gegenübertragungssituation mit suizidalen Patienten reinszeniert werden. Bisweilen kann es auch interaktionell zu einer paradoxen Situation kommen, wenn Therapeut und Patient quasi in einem gemeinsamen unbewussten Abwehrverhalten in einem stillschweigenden Übereinkommen die Suizidalität - als gemeinsam erlebt - verleugnen und auf scheinbar ungefährlichere Themen ,,verschieben“. Wenn der Analytiker sich diese Situation nicht bewusst machen kann, dann darf erwartet werden, dass wenig später es zu einer heillosen „Verstrickung“, zu massiven Missverständnissen und schlimmstenfalls zum Therapieabbruch bis hin zu Suizidversuchen und zu einem Suizid kommen kann.

In den psychiatrischen Kliniken können wir viele negative Gegenübertragungsphänomene beobachten. Hier bemerken wir - insbesondere bei sehr hierarchisch strukturierten Kliniken - einen allen Beteiligten unbewussten institutionellen kollektiven Gegenübertragungswiderstand gegenüber suizidalen Patienten, wie wir ihn sonst am ehesten gegenüber sozial schwachen Alkoholikern beobachten können.

Anfang der 1980er-Jahre waren diese Phänomene an der Hamburger Psychiatrischen Universitätsklinik (und, wie mündlich berichtet, an vielen anderen psychiatrischen Kliniken auch) sehr eindrücklich zu beobachten. Wenn in den täglichen Klinikkonferenzen ein Assistent über einen Suizidversuch oder Suizid eines seiner Patienten berichtete, dann trat regelmäßig ein bedrückendes Schweigen auf, oder es wurden eher wenig relevante Nachfragen gestellt, das Thema wurde rasch gewechselt. Der Assistent blieb mit Schuldgefühlen und dem Gefühl, versagt zu haben, zurück. Auf unsere Initiative hin (P. G.) wurden daraufhin hierarchiearme Suizidkonferenzen eingerichtet, die personell nach den Wünschen des jeweiligen Assistenten zusammengestellt wurden und vorurteilsfrei, kollegial und sehr konstruktiv arbeiteten.

Diese erfolgreiche Arbeit, die sich auf die Ambulanz ausdehnte, motivierte (P. G.), eine ambulant tätige niedrigschwellige Anlaufstelle ausschließlich für suizidgefährdete Patienten einzurichten, zumal zu beobachten war, dass viele suizidale Patienten sich nicht als krank definierten und von daher auch eine stationäre Aufnahme scheuten (O-Ton: „Ich wollte doch nicht sterben, es war doch nur eine Kurzschlussreaktion, jetzt ist es wieder vorbei, ich will nach Hause“").

Durch eine sehr große Spende konnte in einem extra gebauten Pavillon - ohne baulichen Zusammenhang mit der Psychiatrischen Klinik - das Therapie- und Forschungszentrum für Suizidgefährdete (TZS) eingerichtet werden. Unter dem Aspekt, dass die Psychoanalyse auf dem Gebiet der Suizidologie sowohl theoretisch als auch psychotherapeutisch große Erfahrungen vorweisen konnte, wurde dem TZS das psychoanalytische Denken, Handeln und Forschen zugrunde gelegt. Die Einrichtung war 
in Deutschland (und wohl auch darüber hinaus) einzigartig. Im Jahr 1990 nahm das TZS seine Arbeit auf. Die vorliegenden Ausgabe des Forum der Psychoanalyse verbindet ein Themenheft über die vielfältigen Dimensionen von Suizidalität mit der Darstellung dieses Projekts.

Georg Fiedler stellt gemeinsam mit wesentlichen Mitarbeitern des TZS zunächst die Geschichte, Ansätze und die Arbeitsweise des sich als Projekt verstehenden Therapiezentrums vor. Er trägt in einer weiteren Arbeit wesentliche Daten und Fakten zum Suizid zusammen, die man als Psychoanalytiker kennen und wissen sollte. Reinhard Lindner zeigt in einem Überblick, wie seine im Umfeld des TZS entstandenen Forschungsarbeiten dem Thema des Suizids bei alten Menschen nachgegangen sind. Benigna Gerisch entfaltet das Thema der ,weiblichen Suizidalität“ und verknüpft intensive therapeutische Erfahrungen mit Überlegungen zum Suizid als Körperinszenierung unter den Bedingungen von gnadenloser Selbstoptimierung. Mark Goldblatt schildert seine Erfahrungen als aus den USA kommender Supervisor.

Psychoanalyse ist mehr als ein Verfahren, das einem ausgewählten Personenkreis in einem geschützten Rahmen mit hoher Zugangsschwelle einen Ort der langfristigen Selbstreflexion zur Verfügung stellt. Ihr Ansatz kann auch in der praktischen Medizin eingesetzt werden, gleichsam an der Front, indem der klinische Zugang insgesamt psychoanalytisch strukturiert wird. Auch das die Menschheitsgeschichte begleitende Phänomen der Suizidalität erweist sich als ein Beziehungsgeschehen und wird damit zum Gegenstand einer psychoanalytischen Untersuchung und Behandlung. Dies in concreto belegt zu haben, bleibt das Verdienst des TZS. Es wurde ein klinischer Fortschritt markiert, aus dem weiter viel Gutes entstehen könnte: forschende psychoanalytische Einheiten, die einem konkreten Ziel verpflichtet sind: Schafft zwei, drei viele TZSs!

\section{Paul Götze}

\section{Ulrich Lamparter}

Interessenkonflikt P. Götze und U. Lamparter geben an, dass kein Interessenkonflikt besteht.

\section{Literatur}

Bloom V (1967) An analysis of suicide at a training center. Am J Psychiat 123:918-925

Goethe JW (1964) Dichtung und Wahrheit, 13. Buch, 5. Auflage. Goethes Werke, Hamburger Ausgabe in 14 Bänden, Band 9, Christian Wegner Verlag, Hamburg, S 583

Litman RE (1965) When patients commit suicide. Am J Psychother 19:570-576

Maltsberger JT, Buie DH (1974) Countertransference hate in the treatment of suicidal patients. Arch Gen Psychiat 30:625-633

Platon (1990) Des Sokrates Apologie. Platon, Werke in 8 Bänden, zweisprachige Sonderausgabe, Band 2, Wiss. Buchgesellschaft, Darmstadt, S 1-69

Prof. Dr. Paul Götze Von 1982 bis 2007 Professur für psychodynamische Psychiatrie und Psychotherapie. Initiator und Leiter des Therapie- und Forschungszentrums für Suizidgefährdete (TZS) am Universitätsklinikum Hamburg-Eppendorf (1990-2007). Psychoanalytiker (DPV, IPA). Seit 2007 psychoanalytischpsychotherapeutische Privatpraxis.

PD Dr. Ulrich Lamparter Dr. med. Dipl.-Psych., Psychoanalytiker (DPV). Nach langjähriger Tätigkeit an der Poliklinik für Psychosomatische Medizin und Psychotherapie des Universitätsklinikums HamburgEppendorf in eigener Praxis tätig. Arbeitsschwerpunkte: klinische Psychosomatik, Ausbildungsforschung, Kriegskindheit und familiäre Weitergabe traumatischer Erfahrungen. Mitherausgeber dieser Zeitschrift. 